\title{
Routine Surveillance of Chemotherapy Toxicities in Cancer Patients Using the Patient-Reported Outcomes Version of the Common Terminology Criteria for Adverse Events (PRO-CTCAE)
}

\author{
Tian Qi Wang · Joseph N. Samuel · M. Catherine Brown • Ashlee Vennettilli • Hannah Solomon • \\ Lawson Eng · Mindy Liang · Gursharan Gill · Zahra Merali · Chenchen Tian · Nicholas Y. H. Cheng • \\ Matthew Campbell · Devalben Patel · Ai Xin Liu · Geoffrey Liu · Doris Howell
}

Received: May 9, 2018 / Published online: October 22, 2018

(C) The Author(s) 2018

\section{ABSTRACT}

Introduction: Systematic documentation of chemotoxicities in outpatient clinics is challenging. Incorporating patient-reported outcome (PRO) measures in clinical workflows can be an efficient strategy to strengthen the assessment of symptomatic treatment toxicities in oncology clinical practice. We compared the adequateness, feasibility, and acceptability of toxicity documentation using systematic, prospective, application of the PRO Common Toxicity Criteria for Adverse Events (PROCTCAE) tool.

Methods: At a comprehensive cancer center, data abstraction of electronic health record reviews elucidated current methods and degree of chemotoxicity documentation. Web-based 32-item PRO-CTCAE questionnaires, administered in ambulatory clinics of patients receiving

Enhanced digital features To view enhanced digital features for this article go to https://doi.org/10.6084/ m9.figshare.7072124.

T. Q. Wang · J. N. Samuel · M. C. Brown ·

A. Vennettilli · H. Solomon · L. Eng · M. Liang ·

G. Gill · Z. Merali · C. Tian · N. Y. H. Cheng ·

D. Patel · A. X. Liu · G. Liu (ه) - D. Howell

Princess Margaret Cancer Center, Toronto, ON,

Canada

e-mail: Geoffrey.Liu@uhn.on.ca

M. Campbell

Sackler School of Medicine, Tel Aviv University, Tel Aviv, Israel chemotherapy, captured chemotoxicities and respective severities. Patient telephone surveys assessed whether healthcare providers had addressed chemotoxicities to the patients' satisfaction.

Results: Over a broad demographic of 497 patients receiving chemotherapy, 90\% (95\% CI 84-96\%) with significant chemotoxicities $(n=107)$ reported that their providers had discussed toxicities with them; of these, 70\% received a therapy management change, while among the rest, $17 \%$ desired a change in management. Of patients surveyed, 91\% (95\% CI 82-99\%) were satisfied with their current chemotoxicity management. Clinician chart documentation varied greatly; descriptors rather than numerical grading scales were typically used. Although 93\% of patients were willing to complete the PRO survey, only 50\% thought that it would be acceptable to complete this survey at routine clinic visits.

Conclusion: Use of PRO-CTCAE in routine clinical practice promotes systematic evaluation of symptomatic toxicities and improves the clarity, consistency, and efficiency of clinician documentation; however, methods to improve patient willingness to complete this tool routinely are needed.

Keywords: Cancer; Chemotoxicity; Patient reported outcome; PRO-CTCAE; Side-effects monitoring 


\section{INTRODUCTION}

There is an increasing emphasis on improving the quality of cancer treatment side effects and toxicity management, alongside the need to collect real-world pharmacovigilance data to improve health outcomes and quality of life $[1,2]$. This can be achieved through systematically collecting and evaluating chemotherapyrelated adverse events from a patient-centered perspective [3-5]. Unlike healthcare utilization outcomes (e.g., length of hospital stays, morbidity/mortality) [6, 7], subjective and patientreported toxicities may not be captured in electronic health records. Thus, routine prospective patient-reported adverse event data (outside the context of a clinical trial) is appealing.

Chemotherapy related symptomatic side effects are prevalent in a substantial proportion of patients receiving treatment, with the most prevalent being fatigue (59-91\%), decreased appetite (42-62\%), nausea (28-60\%), vomiting $(17-26 \%)$, and oral mucositis (17-40\%) [4, 8-11]. Distressing symptoms during treatment interfere with daily activities and may adversely affect quality of life [12-15]. Moreover, objective Common Terminology Criteria for Adverse Events (CTCAE) toxicity reporting by clinicians may not always align with patient reports [3, 4, 16-20]. Thus, data capture using patient-reported outcomes (PRO) is becoming a standard of care in many cancer organizations; however, generic PRO measures such as the Edmonton Symptom Assessment System may lack precision in detecting treatment-specific toxicities [21]. The US National Cancer Institute's PRO version of the CTCAE is a library of 124 self-report items representing 78 symptomatic toxicities $[22,23]$. For each symptom, up to three items capture the attributes of frequency, severity, and interference with daily activities. The standard recall period is for the past 7 days. As an adjunct to traditional survival outcomes, inclusion of PROs in routine clinical practice are posited to improve patient's healthrelated quality of life (HRQOL) through enhanced communication and feedback to clinicians for therapeutic side effect management, though such positive effects have not been consistently observed [24, 25]. PROCTCAE was developed for use in cancer clinical trials; however, interest in using PRO-CTCAE in routine clinical cancer care has not been previously examined.

Traditionally, treatment toxicities elicited by healthcare providers tend to grade symptom severity lower, compared to patient self-assessments [16-20, 26, 27]. Use of PRO-CTCAE may result in more precise and reliable data about symptomatic adverse events. Electronic PRO data collected through tablets, mobile phones, or webbased applications can be used to auto-populate clinical notes, trigger notifications to healthcare providers when symptoms are clinically significant, and allow for the systematic collection of data for quality monitoring and pharmacoepidemiologic research (including pharmacovigilance). However, patient acceptability and feasibility remain important questions when introducing new methods of routine data collection, especially the collection of data designed to be used dually for clinical management of patients and as a performance metric for improvement in health outcomes and care quality [28, 29].

A recent systematic review suggested that well-implemented PRO tools, particularly when there is systematic attention to provider-feedback and alerts for severe symptoms, may improve patient-provider communication and patient satisfaction, though salutary effects of PROs on patient-centered and health service outcomes are not observed consistently in randomized trials [30]. There is also growing evidence that PRO tools improve the monitoring of treatment response and the detection of unrecognized problems [31, 32]. However, there is a gap in our current knowledge with respect to the effects on care processes and health outcomes of routine collection of PRO-CTCAE as part of cancer care delivery [25, 33-35].

Systematic collection of chemotherapy toxicities can either come from the healthcare provider or directly from the patient. Since healthcare providers have increasing time pressures, the likelihood of success in introducing additional training to conduct directed toxicity interviews seems low [36]. In contrast, PROs in a patient-centered setting could systematically capture and improve documentation of 
chemotoxicities, potentially streamlining aspects of the clinical encounter; however, there are also concerns that improved collection of toxicity burden can lead to increased burden of managing these toxicities. Nonetheless, a fundamental question is whether PRO tools such as the PRO-CTCAE are even needed or acceptable to patients. Perhaps historical methods (dictations, written progress notes, and review of systems checklists) used by healthcare providers already provide rigorous symptomatic toxicity data.

Our aims for this study are (1) to examine the adequateness of toxicity documentation by historical control methods versus when PROCTCAE is used; (2) to determine whether patients receiving anticancer treatment are satisfied with the current management of their symptomatic toxicities, given the healthcare provider-centered data collection processes currently in use; (3) to compare the adequateness of toxicity documentation when using retrospective electronic health record for data collection, as compared to the systematic and prospective application of the PRO-CTCAE tool; (4) to examine the prevalence and severity distribution of the major chemotherapy toxicities; and (5) to determine the feasibility and acceptability of collecting symptom reports electronically using PRO-CTCAE administered in clinic before chemotherapy visits.

\section{METHODS}

Approval was obtained from the University Health Network Research Ethics Board. All procedures performed in studies involving human participants were in accordance with the ethical standards of the institutional and/or national research committee and with the 1964 Helsinki declaration and its later amendments or comparable ethical standards. Informed consent was obtained from all individual participants included in the study. At the Princess Margaret Cancer Centre (Toronto, Ontario, CA, USA), a comprehensive cancer center, adult outpatients over the age of 18 years old, with solid or hematologic malignancies of any stage and who had received chemotherapy in the past month, were eligible to complete PRO-CTCAE online questionnaires administered on touchscreen tablets in the chemotherapy day suites and clinic waiting rooms. Ineligible patients included those with significant cognitive impairments and those without an acceptable level of English comprehension.

When patients were seen in either the clinic or the chemotherapy daycare unit for routine chemotherapy administration, an English-language, touchscreen tablet survey collected information about patient demographics, along with a health utility assessment through the three-level version of the EuroQol 5-Dimensions instrument (EQ-5D-3L) [37], and PRO-CTCAE elements of the five most common and prevalent chemotherapy toxicities (Table 2). EQ-5D-derived health utilities utilized Canadian conversion values to represent general health status, with lower scores indicating worse HRQOL [38, 39].

For the PRO-CTCAE portion, patients were asked to quantify the frequency, severity, and interference with daily activities domains associated with 12 symptomatic toxicities (total of 32 PRO-CTCAE items), using a five-point Likert scale ("never" to "almost constantly" for frequency, "none" to "very severe" for severity, and "not at all" to "very much" for interference), with a 7-day recall period. Significant toxicities were considered a high (point 4) or very high (point 5) Likert scale rating in any of the three domains, as such ratings are likely to result in clinical interventions.

There were several phases of this study (Fig. 1). In the pilot phase, the focus was on (1) proper implementation of the procedures in clinic (which followed an abbreviated CIHR knowledge-to-action framework to identify barriers, facilitators, obtain stakeholder counsel, and identify potential solutions); (2) assessment of patient acceptability (through a short survey of Likert items). Subsequently, in phase I of the study, the focus was on assessing and confirming the appropriateness of toxicity data being collected by PRO-CTCAE, and assessing the current management of any such toxicities by healthcare providers who had no knowledge of results of PRO-CTCAE. Patients in phase I were told that information from these surveys would not be given to any clinician or healthcare 


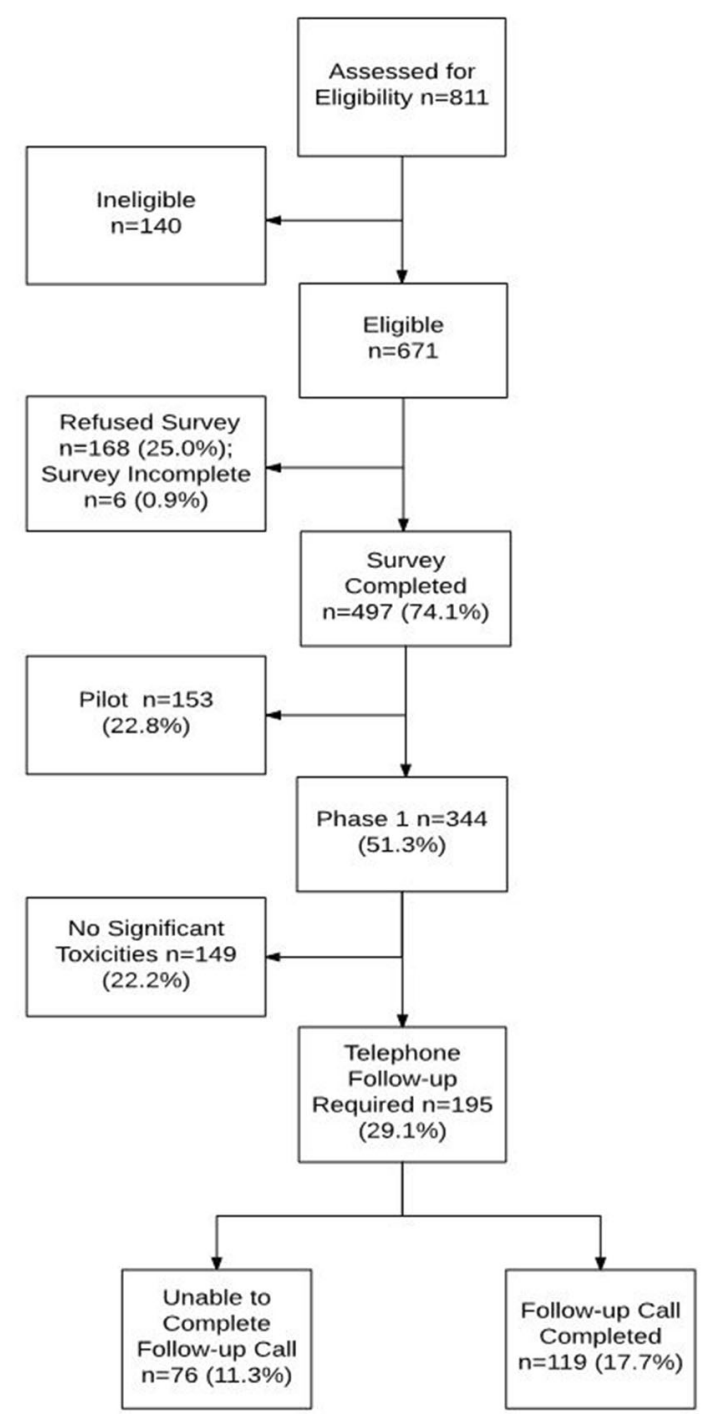

Fig. 1 Consort diagram: summary of recruitment statistics for enrollment into the study

provider, as this represented the pre-intervention period, when we were documenting current practices. There was originally a phase II study whereby PRO-CTCAE results would have been provided immediately to their healthcare providers; improvements in patient care and satisfaction would have been measured relative to phase I. However, phase II was abandoned when phase I demonstrated that patient care and satisfaction was found to be already excellent, even before PRO-CTCAE results were incorporated into the patient management at our institution. Replacing phase II was a retrospective assessment of proper documentation of toxicities in the patient chart record; this was to address a potential secondary reason for incorporating PRO-CTCAE into routine clinical practice, that is, to improve the rate of proper documentation of toxicities, given that proper management of treatment toxicities is an important metric of quality of cancer care.

In phase I, approximately 3 days subsequent to their clinic or day hospital visit (median 3 days; range 1-12), patients were contacted by a member of the non-clinical research team and asked to complete a short telephone survey. The surveys asked whether healthcare providers had discussed toxicities with the patient during their last clinical visit, whether any clinical management changes were instituted as a result of these discussions, and whether patients were satisfied with the outcome of these most recent discussions (Fig. 2). Satisfaction was also collected on a 5-point Likert scale, with " 1 " being very satisfied with care, and " 5 " being very dissatisfied, and dichotomized 1-2 (satisfied) vs 3-5 (neutral or dissatisfied) for analysis.

\section{Phase II Retrospective Assessment of Documentation of Toxicities in the Patient Chart Record}

Data abstraction from the electronic health records confirmed the administration of chemotherapy in the past month, along with any documented symptoms-related toxicities in the electronic pharmacy, nursing, chemotherapy clinic, electronic chemotherapy order entry system, and physician notes within that timeframe. The 497 eligible patients recruited to the study were included in the data abstraction. Chemotoxicity symptoms (to be conservative, any symptom that could be attributed to treatment toxicity was attributed to treatment rather than disease) were recorded along with any corresponding severity rating in its original wording and format. For patients who had been hospitalized, handwritten inpatient notes were also evaluated. For patients enrolled in clinical trials, clinical trial charts were not reviewed, as these were generally not allowed to be used outside the context of the original study. However, all trial primary source data had to be available in the primary electronic charts. 


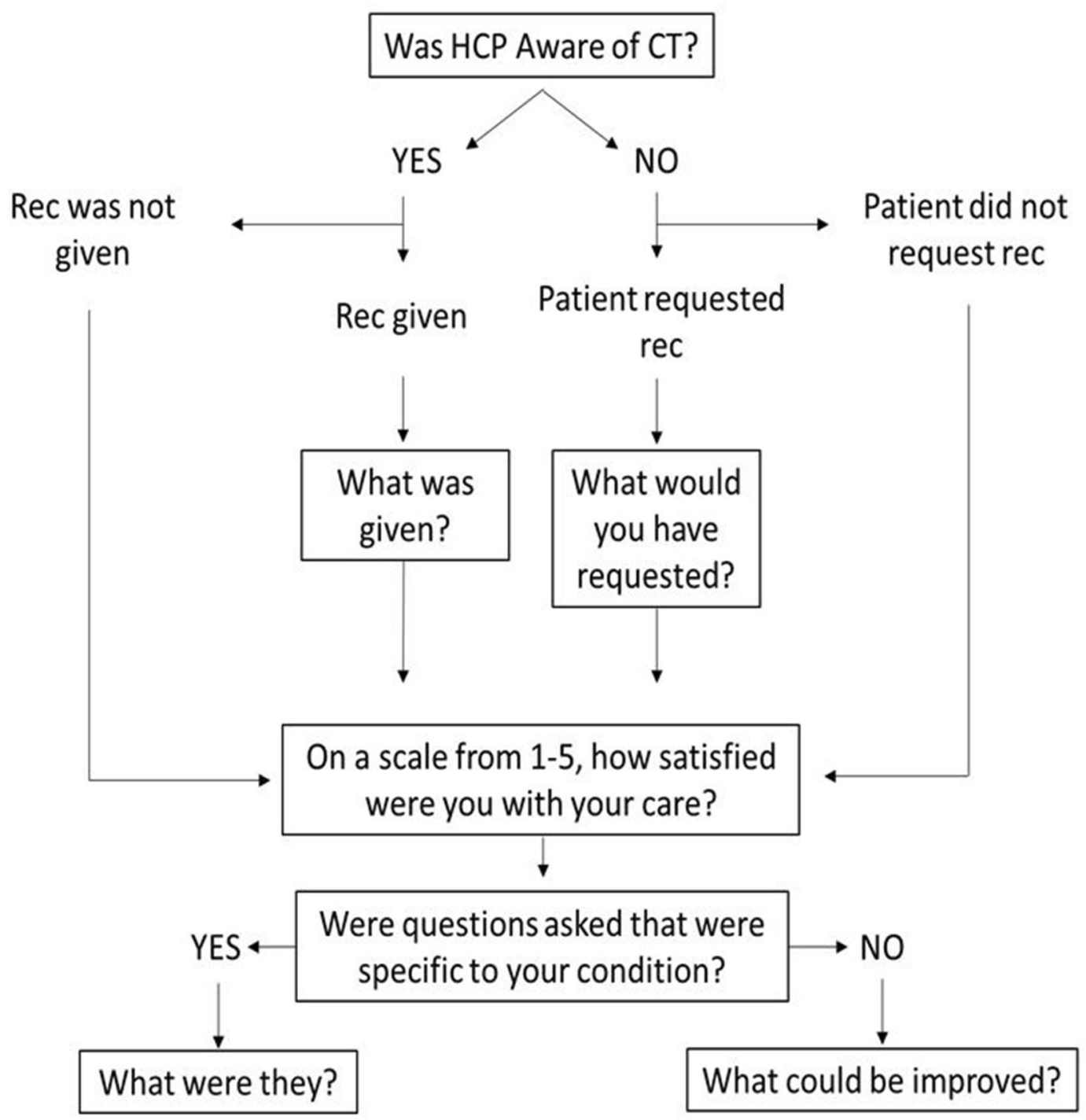

Fig. 2 Flowchart demonstrating the tablet-based survey and follow-up phone survey processes for $n=119$ phase I patients. Prior to using this flowchart, patient toxicities and grades of toxicities were confirmed by the phone

All analyses were performed using SASv9.3.

\section{RESULTS}

\section{Participants}

Of 671 eligible patients, $74 \%(n=497)$ consented and completed PRO-CTCAE and EQ 5D (Fig. 1). Patient clinico-demographic characteristics are shown in Table 1. A diverse range of coordinator. Boxed items are questions that were asked to patients. HCP healthcare provider, CT chemotoxicities, rec recommendation

tumor types and age at diagnosis were represented in this patient population. The median age of recruited patients was 54, with two-thirds being female. PRO-CTCAE was administered to 497: 153 in the pilot phase and 344 in the subsequent phase I portion. Of these 344 patients, 195 reported significant toxicities (defined as having reported at least one score 4 or 5 on any PRO-CTCAE question corresponding to grade 3 or 4 on the CTCAE scales, which are the two highest scores of toxicity for each 
Table 1 Summary of socio-demographic variables from our patient population

\begin{tabular}{|c|c|c|c|}
\hline \multirow[t]{2}{*}{ Variable } & \multirow{2}{*}{$\begin{array}{l}\text { Pilot phase and phase I } \\
\text { PRO-CTCAE administered } \\
\text { in chemotherapy day } \\
\text { hospital or clinic, } N=497\end{array}$} & \multicolumn{2}{|l|}{ Phase I } \\
\hline & & $\begin{array}{l}\text { Patient had any severe symptom as } \\
\text { defined by having at least score } 3 \text { or } 4 \text { on } \\
\text { any PRO-CTCAE item, } N=195\end{array}$ & $\begin{array}{l}\text { Patients reached for } \\
\text { post-outpatient visit } \\
\text { telephone survey, } \\
N=119\end{array}$ \\
\hline $\begin{array}{l}\text { Age (median, } \\
\text { range) }\end{array}$ & $56(18-99)$ & $56(19-85)$ & $54(19-85)$ \\
\hline Gender (\%male) & $42 \%$ & $39 \%$ & $32 \%$ \\
\hline $\begin{array}{l}\text { Ethnicity } \\
\text { (\%Caucasian) }\end{array}$ & $70 \%$ & $74 \%$ & $76 \%$ \\
\hline$\%$ married & $73 \%$ & $68 \%$ & $72 \%$ \\
\hline $\begin{array}{l}\text { \% with any post- } \\
\text { secondary } \\
\text { education }\end{array}$ & $72 \%$ & $74 \%$ & $77 \%$ \\
\hline $\begin{array}{c}\text { \% employed } \\
\text { currently }\end{array}$ & $49 \%$ & $49 \%$ & $50 \%$ \\
\hline \multicolumn{4}{|l|}{ Household income } \\
\hline$<\$ 75 \mathrm{~K}$ & $33 \%$ & $29 \%$ & $30 \%$ \\
\hline $\begin{array}{l}\$ 75 \text { to }<\$ 100 \\
\mathrm{~K}\end{array}$ & $13 \%$ & $13 \%$ & $14 \%$ \\
\hline$\$ 100 \mathrm{~K}+$ & $23 \%$ & $24 \%$ & $22 \%$ \\
\hline \multicolumn{4}{|c|}{ Primary site of disease } \\
\hline Gastrointestinal & $18 \%$ & $9 \%$ & $11 \%$ \\
\hline Genitourinary & $10 \%$ & $12 \%$ & $11 \%$ \\
\hline Gynecological & $12 \%$ & $8 \%$ & $6 \%$ \\
\hline Breast & $19 \%$ & $20 \%$ & $18 \%$ \\
\hline Thoracic & $16 \%$ & $21 \%$ & $18 \%$ \\
\hline Head and neck & $12 \%$ & $15 \%$ & $18 \%$ \\
\hline Hematologic & $13 \%$ & $15 \%$ & $18 \%$ \\
\hline \multicolumn{4}{|l|}{ Intent of treatment } \\
\hline Curative & $35 \%$ & $30 \%$ & $30 \%$ \\
\hline Palliative & $53 \%$ & $55 \%$ & $52 \%$ \\
\hline Hematologic ${ }^{a}$ & $12 \%$ & $15 \%$ & $18 \%$ \\
\hline
\end{tabular}

All values represent percentages of patients except age. The smaller samples represent a subset of the patients in each of the larger datasets

a Hematologic cancers often are not described as either curative or palliative, and thus are listed separately 
question); these patients were to have a followup phone call. Among these 195 patients, 119 received a phone call and successfully completed the phone survey confirming toxicities and management of such toxicities by healthcare providers who were blinded to PRO measures. No interventions were offered during the follow-up phone call, as most chemotoxicities were addressed satisfactorily by in-clinic physician intervention. Major reasons why 76 patients were unable to complete the follow-up phone call survey were three unsuccessful call attempts $(n=35)$, more than 2 weeks had elapsed since PRO survey completion $(n=25)$, missing/wrong phone number $(n=12)$, and other reasons $(n=4)$. The reasons why three phone call attempts were unsuccessful for some patients were mainly logistical. Some possible reasons include patients coincidentally not being at home at the time of the call, missed picking up their cell phone and were unable to call back as a result of outgoing hospital numbers being marked as private, or hospitalization that may be cancer/chemotoxicity-related.

\section{Did Healthcare Providers Inquire About and Discuss Significant Toxicity Issues with Patients?}

In the subset of 119 patients who had significant toxicities and who were surveyed over the phone, 91\% (95\% CI 82-99\%) were satisfied with their chemotoxicity management regimen. The most prevalent moderate to severe symptomatic chemotoxicities were fatigue $(85 \% ; n=101)$, decreased appetite (62\%; $n=74)$, pain $(52 \% ; n=62)$, nausea $(50 \%$; $n=60)$, and difficulty tasting food or drink $(47 \% ; n=56)$. Only $10 \%(n=12)$ of patients reported significant toxicities that their healthcare providers were not aware of. A majority of the patients $(n=107$; mean $90 \%, 95 \% \mathrm{CI}$ 84-96\%) reported that their providers were aware of and had discussed all significant chemotoxicities with them. A large majority of these respondents $(n=83$; mean 70\%, 95\% CI $61-79 \%$ ) also received a change in management as a result of these discussions. Although 30\% $(n=36)$ of the respondents did not receive a change in management after discussing their symptomatic toxicities with their provider, only $17 \%(n=6)$ of such patients reported that they would have wanted a change in symptom management.

\section{How Complete is the Documentation of Toxicities?}

As we collected toxicity data in both the pilot and phase I portion of this study, we were able to compare our PRO-CTCAE data with chart documentation in all 497 patients; 51\% reported at least one significant toxicity of the "severe" or "very severe" grading. In only $34 \%$ of all charts reviewed, chemotoxicities were mentioned in the electronic outpatient clinic charts, and mostly through outpatient physician notes. Documentation format for chemotoxicities varied greatly among providers, and various method of severity scoring were employed. Most providers used qualitative descriptors (e.g., "significant", "some", "slight", "a little bit", and "fair bit") in place of a numerical grading system such as the CTCAE version 4. Quantitative severity measures (e.g., CTCAE) were rarely used (under $10 \%$ of the time).

In contrast, the presence of any chemotoxicities was documented in only $13 / 497$ patients (3\%) in the computerized order entry system: seven patients had specific side effects documented, while another six patients had "patient tolerated treatment well" and similar descriptions in place of a numerical grading system. Adverse events mentioned included creatinine increase $(n=1)$, hypersalivation $(n=1)$, rash $(n=1)$, nausea $(n=2)$, fatigue $(n=1)$, constipation $(n=2)$, and diarrhea $(n=2)$.

\section{How Prevalent Were Symptomatic Adverse Events as Captured by PRO- CTCAE?}

The PRO tool was able to document a high prevalence of the most common symptoms (which we attributed conservatively to chemotoxicities), as well as their severities. In all 497 patients surveyed, $51 \%$ reported at least one grade 3 or higher toxicity (i.e., answered " 4 " or 
"5" on the Likert scales). Among 344 phase I patients, a slightly higher 195 patients or $57 \%$ reported at least one significant grade 3 or higher toxicity in the 7 days prior to PROCTCAE assessment. In the subgroup of 119 patients with at least one significant toxicity confirmed by telephone, the most prevalent frequencies of significant chemotherapeutic toxicities were fatigue (67\%), decreased appetite $(40 \%)$, pain $(34 \%)$, nausea $(29 \%)$, and aching joints (29\%) (Fig. 3; Table 2).

\section{Do Patients Find It Acceptable to Use This Electronic Version of the PRO-CTCAE to Document Their Chemotherapy Adverse Events?}

Patients undergoing the feasibility pilot $(n=153)$ generally had no problems using the touchscreen tablet-administered PRO tool (Table 3). We identified very few barriers, and had no significant problems with implementation. The most frequent barrier that arose was internet disconnection, but these were resolved with refreshment of the application screen and reconnection with the Wi-Fi. Over $80 \%$ of patients reported that questions included in the survey were appropriate and relevant, and were happy to complete the survey using tablet touchscreen technology (Table 3). From qualitative patient assessments, major reasons for not wanting to complete surveys regularly included inconvenience and time requirements in the face of competing requests for other surveys, feeling unwell, and lack of perceived benefit to patient; the last reason was expected since we had told every patient that we would not be relaying this information back to the healthcare treating team of physicians, nurses, and pharmacists as part of this pilot phase.

\section{DISCUSSION}

Outside the context of a clinical trial, current documentation of chemotoxicities at our institution was poor, and comparison of the data collected in a systematic manner suggested that clinician documentation does not fully reflect symptoms from the patients' perspective. Although patients reported to be overwhelmingly satisfied with their current clinical care, they continue to experience chemotoxicity-related symptoms that are inadequately documented, thus highlighting the limitations in using patient satisfaction as a surrogate marker for measuring quality of care and health outcomes. The PRO-CTCAE appears to be an easy and effective way of integrating patient selfassessments into current electronic health record systems, to help improve the lack of documentation for chemotoxicities while facilitating its management. PRO results can easily inform healthcare providers of toxicities and elicit early interventions or assessments when necessary, with the future potential to expand into real-time toxicities monitoring for patients at home. With real-time toxicities monitoring, the tool can help mitigate recall bias for patients who have difficulty recalling the frequency and severity of their symptoms between visits $[40,41]$. The tool may also help improve communication between patients and physicians, by reducing the effects of potential barriers such as perceived status differences, language, and patient not feeling well. There is recent data demonstrating a positive impact on patients' HRQOL when a PRO tool was administered for symptoms monitoring on a regular basis both in clinic and at home [42].

This tool can also help facilitate interprofessional communication and collaboration, by actively involving an interdisciplinary team of physicians, nurses, and pharmacists in the monitoring of adverse drug reactions through assessment of survey results. When used in real time, instead of the current pilot method, PROCTCAE may be able to track the onset and progression/amelioration of adverse drug reactions with respect to the timing of drug administration and toxicities management. This offers a proactive reporting model that helps to mitigate the gross underreporting, latency, and inconsistency that come with the traditional, more passive reporting systems as demonstrated in this study. In the setting of quality assessment studies, PRO-CTCAE-collected adverse reaction information can eliminate the need for retrospective data abstraction, 


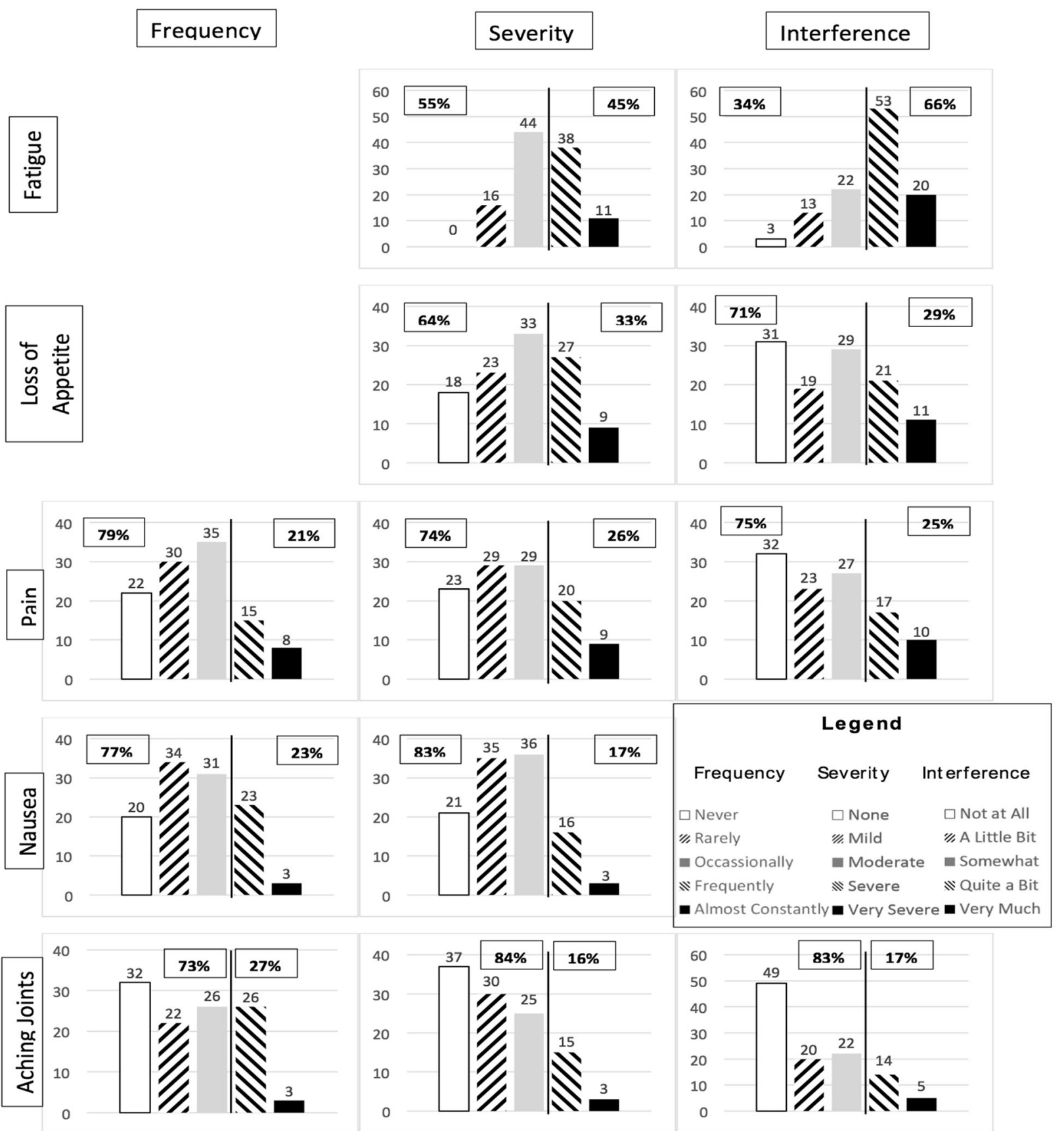

Fig. 3 Prevalence of individual chemotoxicities (at various stage of treatment) as captured by PRO-CTCAE. Each bar represents the frequency of a level (see legend). Numbers reported above each bar represent the number of individuals reporting that level for each symptom. Bars to the right of the solid black line indicate significant toxicity. Percentage of significant and non-significant sums are represented in the percentage boxes on each side of the graphs. "Never/None/Not at all" is reported as "grade 1" in the text, and the extreme "almost constantly/very severe/ very much" is reported as "grade 5". Some symptoms are missing either frequently, severity, or interference, and this was because the PRO-CTCAE items determined that these questions were not as relevant to the symptom assessment 
Table 2 Prevalence of significant toxicities (at various stages of treatment) as captured by PRO-CTCAE, among all individuals reporting at least one significant toxicity and who had received a follow-up phone call

\begin{tabular}{llll}
\hline Symptoms & Frequency & $\begin{array}{l}\text { Severity } \\
(\%)\end{array}$ & Interference \\
\hline Fatigue & - & 45 & $66 \%$ \\
Loss of appetite & - & 33 & $29 \%$ \\
Pain & $21 \%$ & 26 & $25 \%$ \\
Nausea & $23 \%$ & 17 & - \\
Aching joints & $27 \%$ & 16 & $17 \%$ \\
\hline
\end{tabular}

which requires significant resources to conduct and often faces numerous methodological issues $[43,44]$.

There are a number of limitations for this analysis. Firstly, this study was carried out in a single institution and the findings may not reflect all oncology care settings. Secondly, the act of collecting PRO-CTCAE data may have influenced subsequent ratings of satisfaction by participants, whereby participants may have subconsciously considered the collection of such data (with no reporting to their healthcare providers) as an intervention. By completing the PRO questionnaire prior to their clinic visit, patients may have also been prompted to discuss their chemotoxicities with physicians when they would not have otherwise. Chart reviews were conducted using electronic records; however, a limited number of paper charts were reviewed as well and no additional toxicity data were found. Furthermore, the follow-up call was conducted by a research coordinator from the same institution; thus, patients may have responded more positively than had the call been made by an impartial third party. Thirdly, as with any study that relies on chart review to reflect what took place in the clinical encounter, it is possible that these findings under- or overestimate the true occurrence of discussions of symptomatic toxicities. Providers may omit detail in their documentation because of time pressures, or fail to accurately recall the specific issues addressed during the patient's visit. Fourthly, acceptability was measured on the basis of patients' perception of whether or not they would be willing to complete the PRO questionnaire, rather than prospectively collected rates of completion; the fact that no intervention was tied to the acceptability may have resulted in fewer patients willing to accept the tool. Lastly, we did not extensively explore, through either focus groups or in-depth interviews, the reasons why some patients did not want to complete the PRO-CTCAE on a regular

Table 3 Patient acceptability of the electronic PRO-CTCAE tool

\begin{tabular}{lllllll}
\hline & $\begin{array}{l}\text { Definitely } \\
\text { not (\%) }\end{array}$ & $\begin{array}{l}\text { Probably } \\
\text { not (\%) }\end{array}$ & $\begin{array}{l}\text { Not } \\
\text { sure (\%) }\end{array}$ & $\begin{array}{l}\text { Probably } \\
\text { yes (\%) }\end{array}$ & $\begin{array}{l}\text { Definitely } \\
\text { yes (\%) }\end{array}$ & $\begin{array}{l}\text { Missing } \\
\text { (\%) }\end{array}$ \\
\hline $\begin{array}{l}\text { Were you happy to complete the surveys on } \\
\text { a touchscreen tablet? }\end{array}$ & 3 & 2 & 6 & 24 & 60 & 5 \\
$\begin{array}{l}\text { Was the completion of the surveys time- } \\
\text { consuming? }\end{array}$ & 55 & 25 & 3 & 9 & 4 & 4 \\
$\begin{array}{l}\text { Did the completion of surveys make your } \\
\text { clinic visit more difficult? }\end{array}$ & 76 & 14 & 3 & 2 & $<1$ & 4 \\
$\begin{array}{l}\text { Did the surveys ask the right questions for } \\
\text { you? }\end{array}$ & 4 & 5 & 12 & 44 & 31 & 4 \\
$\begin{array}{l}\text { Were any of the questions irrelevant to you? } \\
\text { Did you find the questions upsetting or }\end{array}$ & 30 & 22 & 9 & 21 & 14 & 4 \\
distressful? & 77 & 14 & 1 & 2 & $<1$ & 5 \\
\hline
\end{tabular}


basis; there is a need to perform such analyses across multiple institutions in the future.

In summary, although patients were satisfied with the management of their chemotoxicities, documentation for such issues was poor, making retrospective data collection for pharmacovigilance or clinical management assessments difficult. PRO-CTCAE, a tool generally utilized in research, was found to significantly improve the data collection of such chemotoxicities, and was easy for patients to use in a routine clinical setting. This can lead to earlier detection and prevention of chemotoxicities, potentially limiting its progression to more severe stages that can hinder treatment and patients' HRQOL [45-48]. In addition, the PRO tool can be adapted to detect and report side effects related to newly marketed medications, and flag significant toxicities sooner by allowing easy access to more reliable toxicity data.

\section{ACKNOWLEDGEMENTS}

We thank the participants of the study.

Funding. This study was made possible through a grant to ON-PROST from the Ontario Institute for Cancer Research and Cancer Care Ontario. No funding or sponsorship was received for the publication of this article.

Authorship. All named authors meet the International Committee of Medical Journal Editors (ICMJE) criteria for authorship for this article, take responsibility for the integrity of the work as a whole, and have given their approval for this version to be published.

Disclosures. Tian Qi Wang, Joseph N. Samuel, M. Catherine Brown, Ashlee Vennettilli, Hannah Solomon, Lawson Eng, Mindy Liang, Gursharan Gill, Zahra Merali, Chenchen Tian, Nicholas Y.H. Cheng, Matthew Campbell, Devalben Patel, Ai Xin Liu, Geoffrey Liu and Doris Howell have nothing to disclose.
Compliance with Ethics Guidelines. Approval was obtained from the University Health Network Research Ethics Board. All procedures performed in studies involving human participants were in accordance with the ethical standards of the institutional and/or national research committee and with the 1964 Helsinki declaration and its later amendments or comparable ethical standards. Informed consent was obtained from all individual participants included in the study.

Open Access. This article is distributed under the terms of the Creative Commons Attribution-NonCommercial 4.0 International License (http://creativecommons.org/licenses/ by-nc/4.0/), which permits any noncommercial use, distribution, and reproduction in any medium, provided you give appropriate credit to the original author(s) and the source, provide a link to the Creative Commons license, and indicate if changes were made.

\section{REFERENCES}

1. The state of cancer care in America. 2015: a report by the American Society of Clinical Oncology. J Oncol Pract. 2015;11:79-113.

2. Baldo P, De Paoli P. Pharmacovigilance in oncology: evaluation of current practice and future perspectives. J Eval Clin Prac. 2014;20:559-69.

3. Epstein RM, Street RL Jr. Patient-centered communication in cancer care: promoting healing and reducing suffering. Bethesda: National Cancer Institute; 2007.

4. Xiao C, Polomano R, Bruner DW. Comparison between patient-reported and clinician-observed symptoms in oncology. Cancer Nurse. 2013;36:E1-16.

5. Di Maio M, Gallo C, Leighl NB, et al. Symptomatic toxicities experienced during anticancer treatment: agreement between patient and physician reporting in three randomized trials. J Clin Oncol. 2015;33:910-5.

6. Henry DH, Viswanathan HN, Elkin EP, et al. Symptoms and treatment burden associated with cancer treatment: results from a cross-sectional 
national survey in the U.S. Support Care Cancer. 2008;16:791-801.

7. Shabaruddin FH, Chen LC, Elliott RA, et al. A systematic review of utility values for chemotherapyrelated adverse events. Pharmaco Econ. 2013. https://doi.org/10.1007/s40273-013-0033-x.

8. Kangas M, Bovbjerg DH, Montgomery GH. Cancerrelated fatigue: a systematic and meta-analytic review of non-pharmacological therapies for cancer patients. Am Psychol Assoc. 2008;134:700-41.

9. Wang TQ, Brown C, Vennettilli A, et al. Improving quality of care by obtaining patient-reported outcomes (PRO)-CTCAE chemotoxicities using tablet technology in daycare (DC) waiting rooms. J Clin Oncol. 2014;32:165.

10. Lionel D, Christophe L, Marc A, Jean-Luc C. Oral mucositis induced by anticancer treatments: physiopathology and treatments. Ther Clin Risk Manag. 2006;2:159-68.

11. Cleeland CS, Zhao F, Chang VT, et al. The symptom burden of cancer: evidence for a core set of cancerrelated and treatment-related symptoms from the Eastern Cooperative Oncology Group Symptom Outcomes and Practice Patterns study. Cancer. 2013;119:4333-40.

12. Kayl AE, Meyers CA. Side-effects of chemotherapy and quality of life in ovarian and breast cancer patients. Curr Opin Obstet Gynecol. 2006;18:24-8.

13. Hackbarth M, Haas N, Fotopoulou C, et al. Chemotherapy-induced dermatological toxicity: frequencies and impact on quality of life in women's cancers: results of a prospective study. Support Care Cancer. 2008;16:267-73.

14. Ballatori E, Roila F, Ruggeri B, et al. The impact of chemotherapy-induced nausea and vomiting on health-related quality of life. Support Care Cancer. 2006;15:179-85.

15. Cohen L, de Moor C, Eisenberg P, et al. Chemotherapy-induced nausea and vomiting incidence and impact on patient quality of life at community oncology settings. Support Care Cancer. 2006;15:497-503.

16. Fromme EK, Eilers KM, Mori M, et al. How accurate is clinician reporting of chemotherapy adverse effects? A comparison with patient-reported symptoms from the Quality-of-Life Questionnaire C30. J Clin Oncol. 2014;22:3485-90.

17. Laugsand EA, Sprangers MA, Bjordal K, et al. Health care providers underestimate symptom intensities of cancer patients: a multicenter European study. Health Qual Life Outcomes. 2010;8:10.
18. Atkinson TM, Li Y, Coffey CW, et al. Reliability of adverse symptom event reporting by clinicians. Qual Life Res. 2012;21:1159-64.

19. Basch E, Bennett A, Pietanza mc, et al. Use of patient-reported outcomes to improve the predictive accuracy of clinician-reported adverse events. J Natl Cancer Inst. 2011;103:1808-10.

20. Petersen MA, Larsen H, Pedersen L. Assessing health-related quality of life in palliative care: comparing patient and physician assessments. Eur J Cancer. 2006;42:1159-66.

21. Richardson LA, Jones GW. A review of the reliability and validity of the Edmonton symptom assessment system. Curr Oncol. 2009;16:55.

22. Patient-Reported Outcomes Version of the Common Terminology Criteria for Adverse Events (PROCTCAE). (2018, December). Retrieved June 12, 2014, from https://healthcaredelivery.cancer.gov/ pro-ctcae/.

23. Basch EM, Reeve BB, Mitchell SA et al. Development of the National Cancer Institute's Patient-Reported Outcomes Version of the Common Terminology Criteria for Adverse Events (PRO-CTCAE). J Natl Cancer Inst. 2014;106(9). https://doi.org/10.1093/ jnci/dju244.

24. Mooney A. Quality of life: questionnaires and questions. J Health Commun. 2006;11(3):327-41.

25. Kotronoulas G, Kearney N, Maguire R, et al. What is the value of the routine use of patient-reported outcome measures toward improvement of patient outcomes, processes of care, and health service outcomes in cancer care? A systematic review of controlled trials. J Clin Oncol. 2014;32(14):1480-501. https://doi.org/10.1200/ JCO.2013.53.5948.

26. Carle P, Andrew G, Bushmakin J, et al. Do Patients and physicians agree in their assessment of the severity of psoriasis? Insights from tofacitinib phase 3 clinical trials. J Dermatol Clin Res. 2015;3(3):1048. https://doi.org/10.1111/j.13652133.2012.10991.

27. Suarez-Almazor ME, Conner-Spady B, Kendall CJ, et al. Lack of congruence in the ratings of patients' health status by patients and their physicians. Med Decis Mak. 2001;21(2):113-21.

28. Hannah S, Catherine B, Ashlee V, et al. Involving patients to improve their care through real-time patient reported outcome (PRO)-CTCAE chemotoxicity surveys in an outpatient chemodaycare (DC) setting: evaluating patient acceptability. In: 2014 ASCO Quality Care Symposium; 2014 October 17-18; Boston, MA. J Clin Oncol (Abstract nr 64). 
29. Vivien P, Catherine B, Ashlee V, et al. Using tablet technology in routine patient-reported outcome measure surveys to improve cancer quality care: a patient acceptability assessment. ASCO Quality Care Symposium; 2014 October 17-18; Boston, MA. J Clin Oncol (Abstract nr 155).

30. Chen J, Ou L, Hollis SJ. A systematic review of the impact of routine collection of patient reported outcome measures on patients, providers and health organisations in an oncologic setting. BMC Health Serv Res. 2013;13:211. https://doi.org/10. 1186/1472-6963-13-211.

31. Velikova G, Booth L, Smith AB, et al. Measuring quality of life in routine oncology practice improves communication and patient well-being: a randomized controlled trial. J Clin Oncol. 2004;22:714-24. https://doi.org/10.1200/JCO.2004.06.078.1.

32. Howell D, Molloy S, Wilkinson K, et al. Patient-reported outcomes in routine cancer clinical practice: a scoping review of use, impact on health outcomes, and implementation factors. Ann Oncol. 2015. https://doi.org/10.1093/annonc/mdv181.

33. Mooney KH, Beck SL, Friedman RH, et al. Automated monitoring of symptoms during ambulatory chemotherapy and oncology providers' use of the information: a randomized controlled clinical trial. Support Care Cancer. 2014;22(9):2343-50. https:// doi.org/10.1007/s00520-014-2216-1.

34. Takeuchi EE, Keding A, Awad N, et al. Impact of patient-reported outcomes in oncology: a longitudinal analysis of patient-physician communication. J Clin Oncol. 2011;29:2910-7. https://doi.org/10. 1200/JCO.2010.32.2453.

35. Nama V, Nordin A, Bryant A. Patient-reported outcome measures for follow-up after gynaecological cancer treatment. Cochrane Database Syst Rev. 2013;11:CD010299. https://doi.org/10.1002/ 14651858.cd010299.

36. Zon RT, Frame JN, Neuss MN, et al. American Society of Clinical Oncology policy statement on clinical pathways in oncology. J Oncol Pract. 2016;12(3):261-6. https://doi.org/10.1200/JOP. 2015.009134 .

37. Rabin R, Charro F. EQ-5D: a measure of health status from the EuroQol Group. Ann Med. 2001;33(5):337-43.

38. Agency for Healthcare Research and Quality. U.S. Valuation of the EuroQol EQ-5D Health States; January 2012. http://archive.ahrq.gov/ professionals/clinicians-providers/resources/rice/ EQ5Dproj.html. Accessed 20 Oct 2016.

39. Sullivan PW, Ghushchyan V. Mapping the EQ-5D index from the SF-12: US general population preferences in a nationally representative sample. Med Decis Making. 2016;26:401-9.

40. Giesinger JM, Wintner LM, Zabernigg A, et al. Assessing quality of life on the day of chemotherapy administration underestimates patients' true symptom burden. BMC Cancer. 2014;14:758. https://doi.org/10.1186/1471-2407-14-758.

41. Coolbrandt A, Heede KV, Vanhove E, et al. Immediate versus delayed self-reporting of symptoms and side effects during chemotherapy: does timing matter? Eur J Oncol Nurs. 2011;15(2):130-6. https://doi.org/10.1016/j.ejon.2010.06.010.

42. Basch E, Deal AM, Kris MG, et al. Symptom monitoring with patient-reported outcomes during routine cancer treatment: a randomized controlled trial. J Clin Oncol. 2015. https://doi.org/10.1200/ jco.2015.64.9491.

43. Gilbert EH, Lowenstein SR, Koziol-McLain J, et al. Chart reviews in emergency medicine research: where are the methods? Ann Emerg Med. 1996;27:305-8.

44. Vassar M, Holzmann M. The retrospective chart review: important methodological considerations. J Educ Eval Health Prof. 2013;10:12. https://doi.org/ 10.3352/jeehp.2013.10.12.

45. Young JS, Simmons JW. Chemotherapeutic medications and their emergent complications. Emerg Med Clin N Am. 2014;32(3):563-78. https://doi. org/10.1016/j.emc.2014.04.006.

46. Hwang SY, Chang SJ, Park B-W. does chemotherapy really affect the quality of life of women with breast cancer? J Breast Cancer. 2013;16(2):229-35. https:// doi.org/10.4048/jbc.2013.16.2.229.

47. Tachi T, Teramachi $\mathrm{H}$, Tanaka $\mathrm{K}$, et al. The impact of outpatient chemotherapy-related adverse events on the quality of life of breast cancer patients. PLoS One. 2015;10(4):e0124169. https://doi.org/10. 1371/journal.pone.0124169.

48. Ballatori E, Roila F. Impact of nausea and vomiting on quality of life in cancer patients during chemotherapy. Health Qual Life Outcomes. 2003;1:46. https://doi.org/10.1186/1477-7525-146. 